\title{
DIAGNOSTIC DILEMMA IN INGUINAL HERNIA REPAIR
}

Shreyas $\mathrm{N}^{1}$, Dinesh H. N², Anandravi B. $\mathrm{N}^{3}$

\section{HOW TO CITE THIS ARTICLE:}

Shreyas N, Dinesh H. N, Anandravi B. N. "Diagnostic Dilemma in Inguinal Hernia Repair". Journal of Evolution of Medical and Dental Sciences 2014; Vol. 3, Issue 31, July 31; Page: 8592-8594,

DOI: $10.14260 /$ jemds/2014/3091

ABSTRACT: Tumors presenting in the inguinal hernia sac are considered to be extremely rare, with the more common neoplasms metastasizing from the gastrointestinal tract, ovary and prostate. We report the case of Non-Hodgkin's lymphoma identified in the inguinal hernia sac following hernia repair. Patient presented with obstructed inguinal hernia and was taken up for emergency surgery. Twelve previous cases of a lymphoma diagnosed during hernia repair have been described in the English literature. This case illustrates the value of routine microscopic evaluation of hernia sacs found from inguinal/femoral herniorrhaphies, as it may be the primary presentation of an asymptomatic metastatic lymphoma. Additionally, it underscores the importance of the surgeon's role in screening hernia sacs if the practice of submitting only macroscopically abnormal specimens for microscopic evaluation is adopted.

KEYWORDS: Nonhodgkins lymphoma, inguinal hernia.

INTRODUCTION: Malignant masses in inguinal hernias appear in less than $0.5 \%$ of excised sacs. The tumors can be classified as saccular when they are primary (mesothelioma) or when metastatic disease involves the peritoneal sac itself, or as intrasaccular when the sac contains an organ with a primary malignant mass, as occurred in our case. ${ }^{1}$ At the time of diagnosis, the lymphoma is typically found diffusely throughout the lymphoid tissue, and may be found in other tissues including the intestinal tract, skin and breast. ${ }^{2}$

We present the case of Hodgkin's lymphoma found in inguinal hernia repair, in which histologic evaluation of the hernia sac showed lymphoma. While the pathologist did note irregularity of the hernia sac, no macroscopic abnormality was observed intra operatively. For this patient, routine histologic evaluation of the inguinal hernia sac allowed for the diagnosis and treatment of an aggressive lymphoma that may have otherwise remained undiagnosed.

CASE REPORT: A previously healthy 65year-old male presented with right groin pain and progressive swelling in the inguinal region of several months duration and constipation since one week with no prior history of hernia, heavy lifting or trauma. He reported no other symptoms including fatigue, night sweats or weight loss. Past medical history was insignificant except for being a chronic smoker. Family history was negative for any malignancy.

Vital signs were within normal limits and physical examination was notable only for a rightsided irreducible inguinal hernia which was hard in consistency. Patient was posted for emergency hernia repair and swelling was approached with an inguinoscrotal incision, a hard whitish mass was found which was completely excised. The specimen subjected for histopathological examination and it turned out to be non-Hodgkin's lymphoma. 
DISCUSSION: Hernia sac tumors can be classified into one of three groups: intrasaccular, in which an organ bearing the tumor is incarcerated within the sac; saccular, in which the tumor encompasses the sac or spermatic cord structures; or extrasaccular, in which the tumor protrudes through the hernia defect but is located outside the sac. ${ }^{3}$ Accordingly, the metastatic tumor described in this case can be classified as a saccular hernia sac tumor.

The most common cause of saccular tumors is metastases from abdominal malignancies that migrate under the influence of gravity. ${ }^{3}$ Hernia sac tumors are associated with a wide spectrum of tumor types. Among them, the most frequent primary site is the colon followed by the ovaries, prostate, pancreas, and appendix. ${ }^{4}$

Overall, less than $0.5 \%$ of hernia sacs contain primary or metastatic tumors. ${ }^{5}$ Hernia sac tumors can be suspected when an irreducible, growing mass appears within an inguinal hernia. Since hernia repair offers an opportunity for peritoneal biopsy, selective microscopic examination of grossly abnormal hernia sac specimens is recommended. ${ }^{6}$

\section{REFERENCES:}

1. Tan GY, Guy RJ, Eu KW. Obstructing sigmoid cancer with local invasion in an incarcerated inguinal hernia. ANZ J Surg 2003; 73: 80-2.

2. U.S. Department of Health and Human Services, Center for Disease Control and Prevention, National Center for Health Statistics, author. Health, United States 2007 with Chart book on Trends in the Health of Americans. 2007. pp. 366-367.

3. Nicholson CP, Donohue JH, Thompson GB, Lewis JE. A study of metastatic cancer found during inguinal hernia repair. Cancer 1992, 69:3008-3011.

4. Lowenfels AB, Rohman M, Ahmed N, Lefkowitz M. Hernia-sac cancer. Lancet 1969, 1:651.

5. Yoell JH. Surprises in the hernia sacs. Calif Med. 1959; 91: 146-148.

6. Naoyuki Yokoyama1, Yoshio Shirai, Hidehiro Yamazaki, Katsuyoshi Hatakeyama. An inguinal hernia sac tumor of extrahepatic cholangiocarcinoma origin. World J Surg Oncol. 2006; 4: 13. Published online Mar 6, 2006. doi: 10.1186/1477-7819-4-13.
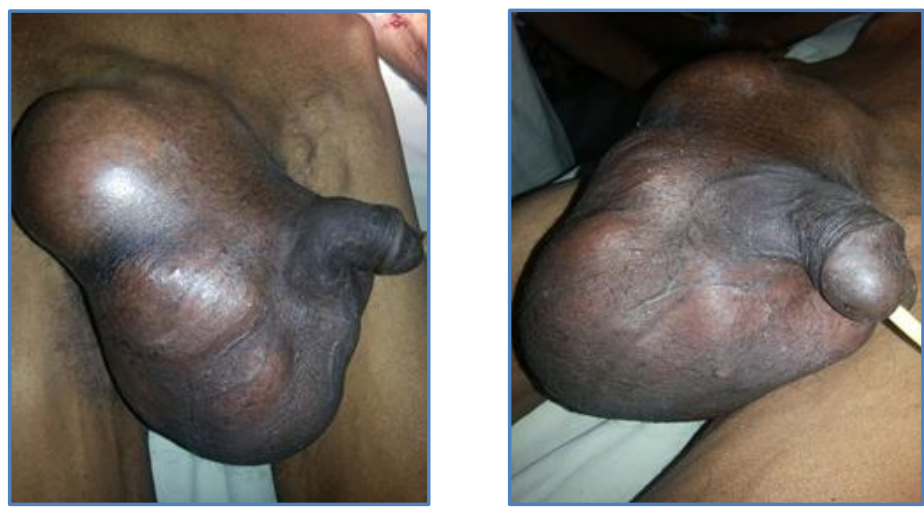

Photo: pre- operative picture of inguinal hernia 


\section{CASE REPORT}
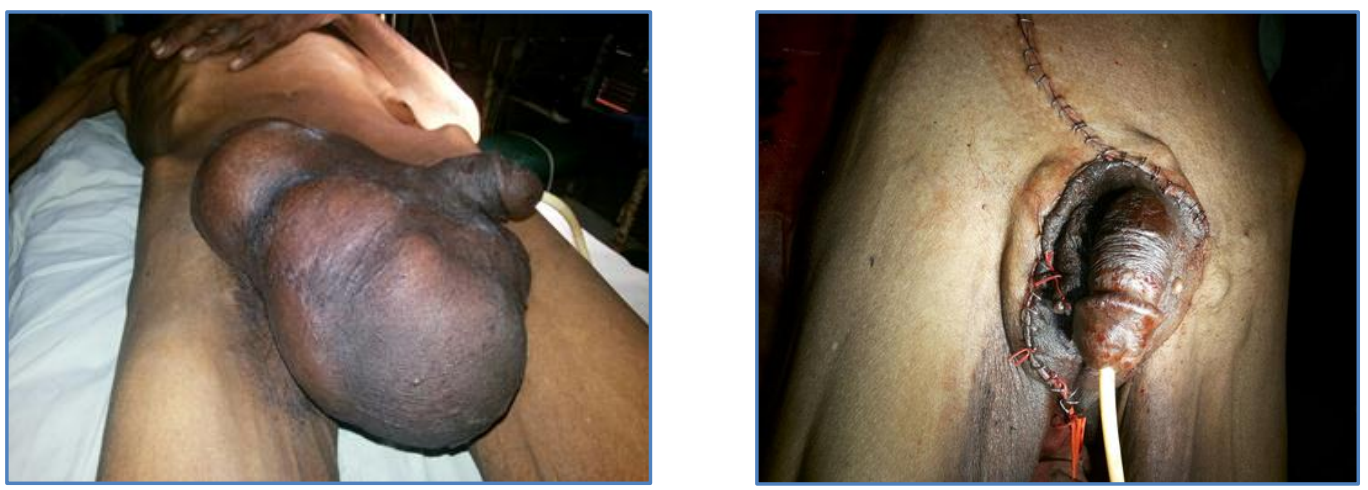

Photo: post-operative picture of hernia repair

\section{AUTHORS:}

1. Shreyas N.

2. Dinesh H. N.

3. Anandravi B. N.

\section{PARTICULARS OF CONTRIBUTORS:}

1. Senior Resident, Department of General Surgery, Mysore Medical College and Research Institute.

2. Associate Professor, Department of General Surgery, Mysore Medical College and Research Institute.

3. Assistant Professor, Department of General Surgery, Mysore Medical College and Research Institute.

\section{NAME ADDRESS EMAIL ID OF THE CORRESPONDING AUTHOR: \\ Dr. Shreyas N, $\# 110,6^{\text {th }}$ Street, Bank Avenue, Babasapalya, Banaswadi, Bangalore-43 \\ Email: doctorshreyas@gmail.com}

Date of Submission: 11/07/2014.

Date of Peer Review: 12/07/2014. Date of Acceptance: 24/07/2014. Date of Publishing: 30/07/2014. 